\title{
Patrones de consumo de drogas en las cárceles en el Perú
}

Patterns of drug use in jails in Peru

Mg. José Luís Colque

Casas

Instituto de Especialización e Investigación en Psicología

Forense y Criminología (IPSIFOC)

Recepción $10 / 06 / 2018$

Revisado 17/07/2018

Aceptación 20/08/2018

\section{RESUMEN}

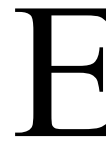
1 presente estudio tuvo como objetivo determinar los patrones de consumo de drogas en sujetos recluidos en un establecimiento penitenciario de Lima. Es un estudio transversal descriptivo y la muestra estuvo conformada por 330 internos varones, de los cuales se pudo validar la información de 279 internos. La edad entre los internos varió desde los 18 a más de 65 años, siendo la edad de mayor prevalencia $(31,2 \%)$ entre 31 a 40 años; el interno con menor edad tiene 18 años y el de mayor edad fue de 75 años. Se utilizó un cuestionario de opciones múltiples, adaptados para la presente investigación, la cual está dividida en cinco áreas: a) nivel educativo, b) estado civil, c) situación jurídica, d) delito y e) consumo de drogas. Asimismo se utilizó el cuestionario de uso de Drogas (DAST20), cuya confiabilidad varía entre un 0,92 a 0,94 . También se utilizó el cuestionario de Identificación de Trastornos por consumo de alcohol (AUDIT), cuyo nivel de confiabilidad se situaba por encima de 0,90. Entre las conclusiones más importantes destacan que las drogas de mayor prevalencia entre las personas recluidas son el alcohol, tabaco, marihuana, clorhidrato de cocaína y pasta básica de cocaína, siendo estas mismas las de mayor consumo en los últimos 30 días. La edad, el grado de instrucción y el delito influyen significativamente en el consumo de drogas al interior del centro penitenciario. 
Palabras claves: alcohol, tabaco y drogas, centro penitenciarios.

\section{ABSTRACT}

7 he objective of this study was to determine patterns of drug use in subjects held in a 1 penitentiary in Lima. It is a descriptive crosssectional study and the sample consisted of 330 male inmates, from whom the information of 279 inmates could be validated. The age among the inmates varied from 18 to over 65 years, being the age of highest prevalence $(31.2 \%)$ between 31 to 40 years, the intern with younger age is 18 years and the oldest age was 75 years. A questionnaire of multiple options was used, adapted for the present investigation, which is divided into five areas: a) educational level, b) civil status, c) legal situation, d) crime and e) drug consumption. Likewise, the Drug Use Questionnaire (DAST-20) was used, whose reliability varies between 0.92 to 0.94 . The Alcohol Disorders Identification Questionnaire (AUDIT) was also used, whose level of reliability was above 0.90 . Among the most important conclusions, the most prevalent drugs among the inmates are alcohol, tobacco, marijuana, cocaine hydrochloride and basic cocaine paste, these being the ones with the highest consumption in the last 30 days. Age, level of education and crime significantly influence drug use inside the penitentiary.

Keywords: alcohol, tobacco and drugs, correctional facility 


\section{Introducción}

La dependencia y abuso de drogas entre la población penitenciaria es una dura realidad que complica enormemente la labor de rehabilitación de las personas privadas de libertad; a esta realidad se suma la grave crisis que vive el sistema penitenciario peruano, donde el hacinamiento es una constante, debido al aumento exacerbado de la población reclusa y al deterioro que vienen sufriendo las instalaciones penales.

Según los diarios, La República (2017), El Comercio (2017) y RPP (2016), en los centros penitenciarios constantemente se realizan reuniones sociales donde el consumo de alcohol y drogas es algo común y cotidiano. Existen mafias que controlan las drogas al interior de las cárceles a pesar de existir vigilancia y continuas detenciones de aquellos que intentan ingresar sustancias prohibidas al interior de la cárcel. Una investigación desarrollada por el diario El País de México (2017), revela en un reportaje con cámara escondida, la venta de drogas delante de las autoridades al interior de una cárcel mexicana.

La población penitenciaria joven es un grupo especialmente vulnerable hacia el consumo de alcohol y drogas; la presencia de estas sustancias implica que muchos reclusos puedan empezar a consumirlas; algunos otros incrementen sus hábitos de consumo y experimenten con nuevas sustancias; pero lo más grave es que muchos de los que consumen muestren conductas más perjudiciales como es el incremento de la agresividad, homicidios por encargo, prostitución y el servilismo y reciben como pago el mantenimiento de su adicción.

El porcentaje de presos dentro de la Unión Europea (2012) que afirman haber consumido alguna vez una droga ilegal varía entre el $29 \%$ y el $86 \%$ (más del $50 \%$ en la mayoría de los estudios), dependiendo de la prisión y el país de que se trate. Al igual que en la sociedad, el cannabis es la sustancia consumida con mayor frecuencia, pero varios estudios muestran elevados niveles de experiencias con heroína (cerca del $50 \%$ de los presos o incluso más en algunos casos).

En el estudio desarrollado por DEVIDA (2013), en el Perú, entre 1,458 adolescentes infractores de la Ley penal, se encontró consumo problemático de alcohol entre un $25 \%$ y un $45 \%$ de los adolescentes infractores. La mitad de los adolescentes infractores han presentado consumo problemático de 
alcohol 30 días antes de cometer la infracción. Siete de cada diez adolescentes infractores presentaron signos de dependencia a PBC, inhalantes, marihuana y alcohol. Seis de cada diez adolescentes infractores consumieron alcohol 30 días antes de haber cometido la infracción y tres de cada diez consumieron marihuana. Uno de cada tres adolescentes infractores había consumido alcohol el mismo día de la comisión de la infracción. Asimismo, uno de cada diez consumió marihuana. Cerca del 5\% había consumido PBC, $2.6 \%$ cocaína y $1.5 \%$ inhalantes el mismo día de la comisión de la infracción. Cuatro de cada diez adolescentes infractores se encontraban bajo efectos de consumo de alguna sustancia, el día que cometieron la infracción. Los delitos de mayor peligrosidad se vinculan en mayor porcentaje con el consumo de alcohol (37.1\%), el día que se cometió la infracción.

En el Estudio Diagnóstico de Salud Mental en establecimientos penitenciarios, desarrollado por el CENECP (2007), se entrevistó a 261 internos a nivel nacional; se encontró que el $64.8 \%$ de las personas recluidas consumían alcohol y que el 19,2\% consumía de forma diaria; en cuanto al consumo de drogas el $50.96 \%$ de la población afirma haber consumido alguna vez en su vida y cerca del $29.12 \%$ afirma haber consumido en el último año y el $18.78 \%$ continúa consumiendo de forma regular.

En un estudio transversal, desarrollado en la Penitenciaria Central de Honduras, cuyos objetivos fueron estimar la tasa de prevalencia o incidencia de posibles trastornos mentales de las personas privadas de libertad, se encontró que las estimaciones correspondían a drogadicción $24.3 \%$, psicosis $20.1 \%$, alcoholismo $13.4 \%$, epilepsia $4.5 \%$, depresión 3.5\% y ansiedad $1.8 \%$.

No obstante el interés en el tema, en el país no se ha realizado una investigación que mida de forma sistemática el consumo de drogas en las personas que están recluidos. Es decir, se afirma la existencia del consumo, pero no se sabe el grado del consumo de drogas en esta población. Se espera con esta investigación llenar este vacío.

El presente estudio parte de la pregunta ¿cuáles son los patrones de consumo de drogas en población recluida en un Establecimiento Penitenciario de Lima?

\section{Métodos}




\section{Diseño}

Se siguió un diseño transversal descriptivo, cuyo objetivo es describir a la población de estudio, sus patrones y características de consumo de drogas; dicho muestreo se realizó en un solo momento. El muestreo fue de tipo no probabilístico. La selección de los sujetos se hizo de forma estratificada según la cantidad de la población recluida en cada pabellón.

\section{Sujetos}

La muestra estuvo constituida por 330 internos varones, de los cuales se pudo validar la información de 279 internos. La edad entre los internos varió desde los 18 a más de 65 años, siendo la edad de mayor prevalencia (31,2\%) entre 31 a 40 años, el interno con menor edad tiene 18 años y el de mayor edad fue de 75 años.

\section{Instrumentos}

Para el presente estudio se diseñó un instrumento tipo cuestionario de opciones múltiples, para el cual se tomó como referencia el cuestionario de estudio nacional de prevención y consumo de droga de DEVIDA, así mismo se utilizó el cuestionario de uso de Drogas (DAST-20), el cual es autoadministrado con respuestas dicotómicas (Sí/No) y diseñado para identificar sujetos con consumo problemático de drogas; su nivel de confiabilidad varía entre un 0,92 a 0,94. También se utilizó el cuestionario de Identificación de Trastornos por consumo de alcohol (AUDIT), que es un instrumento de tamizaje altamente sensible, cuyo nivel de confiabilidad se situaba por encima de 0,90 .

\section{Procedimiento}

La recolección de la información se realizó por pabellones, con autorización del director del establecimiento penitenciario en coordinación con los psicólogos de los diferentes ambientes, quienes facilitaron sus horarios de terapia para desarrollar la presente investigación.

La participación de los internos fue de forma voluntaria. Posterior a la explicación de los objetivos de la presente investigación, algunos manifestaron 
su deseo de participar; mientras otros no desearon hacerlo participar, lo cual se respetó en todo momento.

\section{Resultados}

\section{Características sociodemográficas en relación a su situación legal}

En cuanto a su situación legal, Tabla 1, ante la pregunta ¿cuál es su situación legal en este momento?, más de la mitad de los internos recluidos, respondió, se encuentran en calidad de sentenciados $(72 \%)$, lo cual significa que han sido declarados culpables y un $28 \%$ se encuentra en calidad de procesados o inculpados, esto significa que su situación legal no se encuentra definida.

\section{Tabla 1}

Situación Legal

\begin{tabular}{lcc}
\hline \multicolumn{1}{c}{ Situación Legal } & Frecuencias & Porcentajes \\
\hline Procesados / inculpados & 78 & 28,0 \\
Sentenciados & 201 & 72,0 \\
\hline Total & 279 & $100 \%$ \\
\hline
\end{tabular}

En cuanto a la pregunta ¿cuánto tiempo tiene usted recluido o detenido?, se puede observar en la tabla 2 , que el $46,6 \%$ se encuentran recluido entre 0 a 24 meses, seguidos de los internos con tiempo de reclusión de 25 a 48 meses; el $5,4 \%$ es población que tiene tiempo de reclusión de 73 a 84 meses. El tiempo mínimo de reclusión es menos de un mes y el interno con mayor tiempo de reclusión es de 178 meses lo cual equivale a 14 años 8 meses privados de su libertad.

\section{Tabla 2}


Tiempo de reclusión

\begin{tabular}{|c|c|c|}
\hline Tiempo de reclusión en meses & Frecuencias & Porcentajes \\
\hline $0-24$ & 130 & 46,6 \\
\hline 25 a 48 & 69 & 24,7 \\
\hline 49 a 60 & 22 & 7,9 \\
\hline 61 a 72 & 23 & 8,2 \\
\hline 73 a 84 & 15 & 5,4 \\
\hline 85 a más & 20 & 7,2 \\
\hline Total & 279 & $100 \%$ \\
\hline
\end{tabular}

En la Tabla 3, ante la pregunta ¿por qué delito usted se encuentra?, podemos observar que el 34,4\% se encuentran por delito de tráfico de drogas; seguidos por los internos que cometieron el delito de robo agravado sin violencia $(26,9 \%)$ y en menor porcentaje se encuentran los internos que cometieron el delito de Lesiones $(0,7 \%)$ y Hurto simple $(1,1 \%)$.

Tabla 3

\section{Delitos}

\begin{tabular}{lcc}
\hline Delito & Frecuencias & Porcentajes \\
\hline Robo agravado con violencia & 25 & 9,0 \\
Robo agravado sin violencia & 75 & 26,9 \\
Hurto agravado & 7 & 2,5 \\
Hurto simple & 3 & 1,1 \\
Lesiones & 2 & 0,7 \\
Homicidio & 9 & 3,2 \\
Actos contra el pudor & 22 & 7,9 \\
Violación & 14 & 5,0 \\
Trafico de drogas & 96 & 34,4 \\
Micro comercialización de drogas & 5 & 1,8 \\
Otros & 21 & 7,5 \\
\hline Total & 279 & $100 \%$ \\
\hline
\end{tabular}

\section{Patrones de consumo de drogas}


En la Tabla 4, ante la pregunta, ¿si alguna vez consumió alguna de las siguientes sustancias y a qué edad probó por primera vez?, podemos observar que el $79,9 \%$ de la población estudiada respondió, que consumió alguna vez en su vida alcohol, seguido de consumo de tabaco (62,7\%), marihuana $(42,3 \%)$, clorhidrato de cocaína (27,6\%) y pasta básica de cocaína (20,1\%); un 20,1\% de la población refiere que nunca ha consumido. En menor porcentaje de consumo se encuentra la heroína $(1,4 \%)$.

Con respecto a la edad de inicio de consumo, se puede apreciar que la edad de menor rango es 07 años, relacionada con el consumo de alcohol; 08 años, consumo de inhalantes; 09 años, tabaco y el mayor rango es el de 21 años, relativo al consumo de otras drogas y 20 años relacionado con el consumo de éxtasis. En cuanto a la edad de inicio del consumo de marihuana, cocaína y $\mathrm{PBC}$ se da a los 11 años.

\section{Tabla 4}

Consumo de sustancias y edad de inicio

\begin{tabular}{|l|c|c|c|}
\hline $\begin{array}{l}\text { Alguna vez consumió alguna de las siguientes } \\
\text { sustancias }\end{array}$ & Frecuencias & Porcentajes & Edad de inicio \\
\hline Alcohol & 223 & 79,9 & 07 \\
\hline Tabaco & 175 & 62,7 & 09 \\
\hline Marihuana & 118 & 42,3 & 11 \\
\hline Pasta Básica de Cocaína & 56 & 20,1 & 11 \\
\hline Clorhidrato de Cocaína & 77 & 27,6 & 11 \\
\hline Crack & 12 & 4,3 & 18 \\
\hline Heroína & 4 & 1,4 & 18 \\
\hline Éxtasis & 8 & 2,9 & 20 \\
\hline Inhalante & 20 & 7,2 & 08 \\
\hline Tranquilizante & 39 & 14,0 & 14 \\
\hline Estimulantes & 6 & 2,2 & 16 \\
\hline Alucinógenos & 12 & 4,3 & 11 \\
\hline Otros & 7 & 2,5 & 21 \\
\hline
\end{tabular}

En la Tabla 5, en cuanto a la pregunta ¿cuándo fue la última vez que consumió 
alguna de las siguientes sustancias?, el consumo de alcohol (44,1\%), marihuana $(18,6 \%)$, PBC $(11,1 \%)$ y CC $(13,3 \%)$, refieren haber consumido hace más de un año; en cuanto al consumo de tabaco refieren haber consumido en los últimos 30 días.

Tabla 5

\section{Último consumo}

\begin{tabular}{|l|c|c|c|c|c|c|c|c|}
\hline $\begin{array}{c}\text { Cuándo fue la última } \\
\text { vez que consumió } \\
\text { alguna de las siguientes } \\
\text { sustancias }\end{array}$ & \multicolumn{2}{|c|}{$\begin{array}{c}\text { Nunca he } \\
\text { consumido }\end{array}$} & \multicolumn{2}{|c|}{$\begin{array}{c}\text { Durante los } \\
\text { últimos 30 días }\end{array}$} & \multicolumn{2}{c|}{$\begin{array}{c}\text { Hace más de un } \\
\text { mes pero menos } \\
\text { de un año }\end{array}$} & \multicolumn{2}{c|}{$\begin{array}{c}\text { Hace más de un } \\
\text { año }\end{array}$} \\
\hline N & $\%$ & N & $\%$ & N & $\%$ & N & $\%$ \\
\hline Alcohol & 19 & 6,8 & 26 & 9,3 & 36 & 12,9 & 123 & 44,1 \\
\hline Tabaco & 35 & 12,5 & 68 & 24,4 & 34 & 12,2 & 46 & 16,5 \\
\hline Marihuana & 60 & 21,5 & 18 & 6,5 & 22 & 7,9 & 52 & 18,6 \\
\hline $\begin{array}{l}\text { Pasta Básica de } \\
\text { Cocaína }\end{array}$ & 92 & 33,0 & 7 & 2,5 & 9 & 3,2 & 31 & 11,1 \\
\hline Clorhidrato de Cocaína & 81 & 29,0 & 10 & 3,6 & 11 & 3,9 & 37 & 13,3 \\
\hline Crack & 113 & 40,5 & 1 & 0,4 & 5 & 1,8 & 6 & 2,2 \\
\hline Heroína & 118 & 42,3 & ---- & ---- & 1 & 0,4 & 4 & 1,4 \\
\hline Éxtasis & 115 & 41,2 & ---- & ---- & 2 & 0,7 & 6 & 2,2 \\
\hline Inhalante & 111 & 39,8 & 1 & 0,4 & 1 & 0,4 & 13 & 4,7 \\
\hline Tranquilizante & 96 & 34,4 & 2 & 0,7 & 6 & 2,2 & 25 & 9,0 \\
\hline Estimulantes & 117 & 41,9 & ---- & ---- & 1 & 0,4 & 5 & 1,0 \\
\hline Alucinógenos & 116 & 41,6 & ---- & ---- & 1 & 0,4 & 7 & 2,5 \\
\hline Otros & 118 & 42,3 & ---- & ---- & 1 & 0,4 & 4 & 1,4 \\
\hline
\end{tabular}

En la Tabla 6, ante la pregunta ¿de acuerdo a lo que usted conoce, en su pabellón, cómo es el consumo de las siguientes drogas?, los internos consideran que el consumo de alcohol en su pabellón es un consumo moderado $(11,1 \%)$ junto con el tabaco (18,3\%); de otro lado, consideran que el consumo de marihuana al interior de los pabellones, es uno de los más frecuentes $(10,4 \%)$ junto con la $\operatorname{PBC}(6,1 \%)$, el CC $(7,9 \%)$ y el crack $(1,4 \%)$. 
Tabla 6

De acuerdo a lo que usted conoce, el consumo en los internos es

\begin{tabular}{|c|c|c|c|c|c|c|c|c|}
\hline \multirow{2}{*}{$\begin{array}{l}\text { De acuerdo a lo que usted } \\
\text { conoce en su pabellón, el } \\
\text { consumo es: }\end{array}$} & \multicolumn{2}{|c|}{ Leve } & \multicolumn{2}{|c|}{ Moderado } & \multicolumn{2}{|c|}{ Intenso } & \multicolumn{2}{|c|}{ No consumen } \\
\hline & $\mathrm{N}$ & $\%$ & $\mathrm{~N}$ & $\%$ & $\mathrm{~N}$ & $\%$ & $\mathrm{~N}$ & $\%$ \\
\hline Alcohol & 26 & 9,3 & 31 & 11,1 & 12 & 4,3 & 125 & 44,8 \\
\hline Tabaco & 35 & 12,5 & 51 & 18,3 & 47 & 16,8 & 66 & 23,7 \\
\hline Marihuana & 16 & 5,7 & 17 & 6,1 & 29 & 10,4 & 119 & 42,7 \\
\hline Pasta Básica de Cocaína & 11 & 3,9 & 9 & 3,2 & 17 & 6,1 & 131 & 47,0 \\
\hline Clorhidrato de Cocaína & 12 & 4,3 & 8 & 2,9 & 22 & 7,9 & 128 & 45,9 \\
\hline Crack & 2 & 0,7 & 2 & 0,7 & 4 & 1,4 & 154 & 55,2 \\
\hline Heroína & 2 & 0,7 & 1 & 0,4 & --- & --- & 156 & 55,9 \\
\hline Éxtasis & 2 & 0,7 & 1 & 0,4 & --- & --- & 155 & 55,6 \\
\hline Inhalante & 3 & 1,1 & 2 & 0,7 & --- & --- & 152 & 54,5 \\
\hline Tranquilizante & 6 & 2,2 & 9 & 3,2 & 4 & 1,4 & 141 & 50,5 \\
\hline Estimulantes & 3 & 1,1 & 1 & 0,4 & --- & --- & 147 & 52,7 \\
\hline Alucinógenos & 2 & 0,7 & 1 & 0,4 & --- & --- & 148 & 53,0 \\
\hline Otros & 2 & 0,7 & 2 & 0,7 & --- & --- & 148 & 52,7 \\
\hline
\end{tabular}

\section{Asociación entre las características sociodemográficas y el consumo de drogas en la población recluida}

En la Tabla 7, en cuanto a la relación entre el consumo de tabaco y las variables sociodemográficas (edad, grado de instrucción, estado civil, situación jurídica, tiempo de reclusión y delito), se encontró que existe una relación significativa muy baja, entre la edad y el nivel de consumo de tabaco y es directamente inverso (tiene signos negativo), donde a mayor edad, menor consumo, (rs= $-0,168$ y $\mathrm{p}=0,017)$.

No se encontró una relación significativa, entre el consumo de tabaco y el grado de instrucción ( $\mathrm{rs}=0,143 \mathrm{y} \mathrm{p}=0,044)$, el estado civil $(\mathrm{rs}=-0,065$ y $\mathrm{p}=0,363)$, la situación jurídica $(\mathrm{rs}=0,027 \mathrm{y} \mathrm{p}=0,708)$, el tiempo de reclusión (rs $=-0,077$ y $p=0,277)$ y el delito $(r s=-0,033$ y $p=0,643)$. 
Tabla 7

Regresión logística de consumo de tabaco y variables sociodemográficas. edad, grado de instrucción, estado civil, situación legal, tiempo de reclusión $y$ tipo de delito

\begin{tabular}{lcc}
\hline \multicolumn{3}{c}{ Consumo de Tabaco } \\
\hline \multicolumn{1}{c}{ Variable } & rs & P \\
\hline Edad &,- 168 & 0,017 \\
Grado de Instrucción &, $143^{*}$ & 0,044 \\
Estado civil &, 065 & 0,363 \\
Situación Legal &, 027 & 0,708 \\
Tiempo de reclusión &,- 077 & 0,277 \\
Delito &,- 033 & 0,643 \\
\hline
\end{tabular}

* La correlación es significativa al nivel 0.05 (bilateral)

** La correlación es significativa al nivel 0,01(bilateral)

En la Tabla 8, se presenta la regresión logística entre el consumo de alcohol y las variables sociodemográficas (edad, grado de instrucción, estado civil, situación jurídica, tiempo de reclusión y delito); se muestra que existe una relación significativa muy baja, entre el consumo de alcohol y el tiempo de reclusión ( $\mathrm{rs}=-0,168$ y $\mathrm{p}=0,010)$, y delito ( $\mathrm{rs}=-0,132$ y $\mathrm{p}=0,043)$, siendo ambas directamente inversas (tiene signos negativos), donde a mayor tiempo de reclusión menor consumo de alcohol y en cuanto a delitos de menor gravedad menor consumo de alcohol.

No existe una relación significativa entre el consumo de alcohol, la edad (rs= $-0,109$ y $\mathrm{p}=0,098$ ), grado de instrucción ( $\mathrm{rs}=-0,017 \mathrm{y} \mathrm{p}=0,791$ ), estado civil ( $\mathrm{rs}=0,020$ y $\mathrm{p}=0,757)$, situación jurídica $(\mathrm{rs}=-0,077$ y $\mathrm{p}=0,244)$ y tiempo de reclusión ( $\mathrm{rs}=-0,168$ y $\mathrm{p}=0,010)$. 


\section{Tabla 8}

Regresión logística de consumo de alcohol y variables sociodemográficas. edad, grado de instrucción, estado civil, situación legal, tiempo de reclusión y tipo de delito

\begin{tabular}{lcc}
\hline & \multicolumn{2}{c}{ Consumo de Tabaco } \\
\hline Variable & rs & $\mathrm{p}$ \\
\hline Edad & $-0,109$ & 0.098 \\
Grado de Instrucción & $-0,017$ & 0,791 \\
Estado civil & 0,020 & 0,757 \\
Situación Legal & $-0,077$ & 0,244 \\
Tiempo de reclusión & $-0,168$ & 0,010 \\
Delito & $-0,132$ & 0,043 \\
\hline
\end{tabular}

* La correlación es significativa al nivel 0.05 (bilateral)

** La correlación es significativa al nivel 0,01(bilateral)

La Tabla 9 presenta la regresión logística entre el consumo de drogas y las variables sociodemográficas (edad, grado de instrucción, estado civil, situación jurídica, tiempo de reclusión y delito), la misma muestra la existencia de una relación significativa, muy baja entre la edad y el delito y es directamente inverso (tiene signos negativo), donde a mayor edad, menor consumo, $(\mathrm{rs}=-0,164$ y $\mathrm{p}=0,016)$ y ante delitos de mayor peligrosidad, refleja mayor consumo de drogas $(\mathrm{rs}=-0,250 \mathrm{y} \mathrm{p}=0,000)$. No existe una relación significativa entre el grado de instrucción ( $\mathrm{rs}=-0,107 \mathrm{y} \mathrm{p}=0,117)$, el estado civil ( $\mathrm{rs}=-0,067$ y $\mathrm{p}=0,328)$, la situación jurídica $(\mathrm{rs}=0,038$ y $\mathrm{p}=0,577)$, y el tiempo de reclusión $(\mathrm{rs}=-0,063$ y $\mathrm{p}=0,355)$. 
Tabla 9

Regresión logística de consumo de otras drogas y variables sociodemográficas. Edad, Grado de Instrucción, Estado civil, Situación legal, Tiempo de reclusión y tipo de delito

\begin{tabular}{lcc}
\hline & \multicolumn{2}{c}{ Consumo de Tabaco } \\
\hline Edad &,$- 164^{*}$ & 0,016 \\
\hline Grado de Instrucción &,- 107 & 0,117 \\
Estado civil &, 067 & 0,328 \\
Situación Legal &,- 038 & 0,577 \\
Tiempo de reclusión &,- 063 & 0,355 \\
Delito &,$- 250^{* *}$ & 0.000 \\
Delito & $-0,132$ & 0,043 \\
\hline
\end{tabular}

* La correlación es significativa al nivel 0.05 (bilateral)

** La correlación es significativa al nivel 0,01(bilateral)

\section{Discusión}

El presente estudio encontró que las personas recluidas en un establecimiento penitenciario, en algún momento de su vida consumieron alcohol, tabaco, marihuana, clorhidrato de cocaína y pasta básica de cocaína. En tal sentido, el presente estudio coincide con los realizados por CEDRO y el CENECP, pues la edad de 07 años es la de inicio del consumo de drogas y la edad de inicio tardío fue la edad de 21 años. En cuanto a la pregunta ¿cuándo fue la última vez que consumió alguna sustancia?, cerca de la mitad refiere haber consumido alcohol hace más de un año, junto a la marihuana, clorhidrato de cocaína, pasta básica de cocaína y tranquilizantes. Con respecto al consumo en los últimos 30 días, las drogas mencionadas fueron el tabaco, alcohol, marihuana, clorhidrato de cocaína y pasta básica de cocaína. Asimismo, el estudio encontró que las variables edad y grado de instrucción influyen significativamente en la dependencia hacia el consumo de tabaco, pues se halló una relación significativa inversa. En cuanto a la asociación entre la variable consumo de alcohol, tiempo de reclusión y delitos, existe una relación significativa inversa muy baja; lo cual significa que a menor edad, mayor consumo de alcohol y mayor propensión a delitos como robo agravado, 
violación y tráfico de drogas; no se encontró relación con las variables edad, grado de instrucción, estado civil y situación legal. En cuanto al consumo de drogas y la edad, se encontró que existe una relación significativa inversa muy baja. Por un lado, entre la asociación consumo de drogas - delito, existe una relación significativa inversa baja, esto significa que a mayor edad, menor consumo de drogas. Por otro lado, en cuanto a la relación consumo de drogas - delito, existe una relación significativa inversa baja en los delitos robo agravado, violación y tráfico de drogas. No existe relación entre las variables consumo de drogas, grado de instrucción, estado civil, situación legal y tiempo de reclusión.

\section{Conclusiones}

- Con respecto a las características sociodemográficas encontradas, más de la mitad de la población recluida se encuentra entre los 21 a 40 años.

- En cuanto a la situación legal, más de la mitad de la población estudiada se encuentra en calidad de sentenciados. Referente a los delitos de mayor prevalencia son tráfico de drogas y robo agravado sin violencia.

- Las drogas que mayor prevalencia han tenido en la vida entre los internos recluidos son el alcohol, tabaco, marihuana, clorhidrato de cocaína y pasta básica de cocaína, siendo la edad de inicio los 07 años en el consumo de alcohol y la edad de 11 años en el inicio de consumo de marihuana, clorhidrato de cocaína y pasta básica de cocaína.

- En cuanto al tiempo de consumo, más de la mitad de la población refiere haber consumido hace más de un año alcohol; con respecto al consumo en los últimos 30 días, las drogas ingeridas fueron el alcohol, tabaco, marihuana y clorhidrato de cocaína. Por otra parte, la edad y el grado de instrucción influyen significativamente en la dependencia al consumo de tabaco y alcohol, a menor edad y menor grado de instrucción mayor consumo.

- El tiempo de reclusión y los delitos de robo agravado, violación y tráfico de drogas influyen significativamente en el consumo de alcohol, a menor tiempo de reclusión mayor consumo de alcohol. Asimismo, se encontró que la edad y el delito de robo agravado, violación y tráfico de drogas influyen significativamente en el consumo de drogas, a menor edad mayor consumo de drogas. 


\section{Referencias}

Alvarado, R. (2012). Perspectiva histórica y problemas actuales de la institución penitenciaria en España. Las mujeres encarceladas toman la palabra. (Tesis Doctoral Universidad de Salamanca). Recuperado de http://gredos.usal.es/jspui/bitstream/10366/115530/1/DSC_Alvarado_ Sanchez_Ruth_PerspectivaHistoricaProblemasActuales.pdf.

Beauregard, Luis (13 de Enero 2017). Así se venden y consumen drogas en el Reclusorio Norte de México. El País, México. Recuperado de https:// elpais.com/internacional/2017/01/12/mexico/1484204369_254066. html.

Becoña, E. (2008). Drogodependencias. En Belloch, A., Sandin, B., y Ramos, F., Manual de Psicopatología. Tomo I (pp 375 -402). España: McGrawHill.

Becoña, E., y Cortés, M. (2010). Manual de adicciones para psicólogos especialistas en psicología clínica en formación. Valencia: Socidrogalcohol.

Beltrán, M. (2007). Tratado sobre conductas adictivas. Recuperado de http:// mabeltran.com/Tratado_sobre_conductas_adictivas.pdf.

Cartas de presos revelan cártel de drogas en centros penitenciarios. (16 de abril, 2013). La República. Recuperado de https://larepublica.pe/ archivo/704852-cartas-de-presos-revelan-cartel-de-droga-en-centrospenitenciarios.

Casas, M., Bruguera, E., Duro, P., y Pinet, C. (2011). Conceptos básicos en trastornos adictivos. En Bobes, J., Casas, M. y Gutierrez, M. (Eds), Manual de Trastornos Adictivos (pp. 17 - 26). Madrid: Enfoque.

CEDRO (2002). Prevalencia del consumo de drogas en el Establecimiento Penitenciario del Callao. Lima: CEDRO.

CENECP (2007). Diagnóstico de salud mental en internos de establecimientos penitenciarios de Lima. Lima: INPE.

Comisión Nacional para el Desarrollo y Vida sin Drogas - DEVIDA (2013). 
I Encuesta Nacional sobre el Consumo de Drogas en Adolescentes Infractores del Poder Judicial. Recuperado de http://www.hablafranco. gob.pe/images/upload/descarga/20140722165609I_ENCUESTA_ NACIONAL_CONSUMO_DE_DROGAS_INFRACTORES.pdf.

De Lucas, G (2014). Factores de riesgo y protección del consumo de drogas en menores protegidos de la Comunidad de Madrid. (Tesis doctoral, Universidad Complutense de Madrid). Recuperada de http://eprints. ucm.es/24522/1/T35211.pdf.

En manos de fiscalía quedó suerte de mujer que iba a ingresar drogas en papas. (20 de marzo del 2016). RPP noticias. Recuperado de: https:// rpp.pe/peru/lambayeque/en-manos-de-fiscalia-quedo-suerte-de-mujerque-iba-a-ingresar-droga-en-papas-noticia-946514.

European Monitoring Centre for Drugs and Drug Addiction [EMCDDA]. (2012). Prisons and drugs in Europe: the problem and responses. Recuperado de http://www.emcdda.europa.eu/attachements.cfm/ att_191812_EN_TDSI12002ENC.pdf.

Galindo, J. (2012) Vulnerabilidad en salud de presos del Penal de Villahermosa, Cali, Colombia. (Tesis de Licenciatura Universidad ICESI). Recuperado de https://bibliotecadigital.icesi.edu.co/biblioteca digital/ bitstream/10906/65522/5/vulnerabilidad_salud_presos.pdf.

Graña, J.L. (1994) Conductas Adictivas. Teoría, Evaluación y Tratamiento. Madrid: Debate.

Guardia, J., Surkov, S.I y Cardus, M. (2011). Bases neurobiológicas de la adicción. En Bobes, J., Casas, M. y Gutierrez, M. (Eds), Manual de Trastornos Adictivos (pp. 27 - 35). Madrid: Enfoque.

Hasbún, J., (2002). Estudio de prevalencia y patrones de consumo de drogas en las cárceles dominicanas. Consejo Nacional de Drogas. Dirección de Información e Investigación. Recuperado de http:/odd.gov.do/ Investigaciones/Consumo $\% 20 \mathrm{de} \% 20 \mathrm{drogas} \% 20 \mathrm{en} \% 20$ carceles.pdf.

Iñaki M., y Iñigo, C. (2012). Guía. Atención y tratamientos en prisión por el uso de drogas. España. OME. 
Lorenzo P., Ladero J.M., Leza J.C., Lizasoain I, (2003). Drogodependencias,

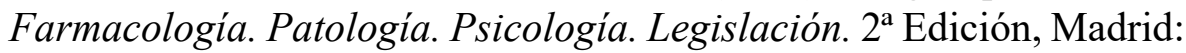
Panamericana.

Moreno, R, Vera-Remartínez, E., García-Guerrero, J., y Planelles M (2008). Consumo de drogas al ingreso en prisión: comparación entre población española y extranjera. Revista Española de Sanidad Penitenciaria; 10: 41. Recuperado de http://scielo.isciii.es/pdf/sanipe/v10n2/original2. pdf.

Morrinson, J. (2015). Guía para el diagnóstico clínico. México: Manual Moderno.

Mumola, C. y Karberg, J. (2006). Drug Use and Dependence, State and Federal Prisoners, 2004. Drug Use and Dependence, State and Federal Prisoners, 2004. U.S. Department of Justice Office of Justice Programs. Recuperado de http://www.bjs.gov/content/pub/pdf/dudsfp04.pdf.

Nevid, J (2012). Essentials of Psychology Concepts and Applications. USA: Cengage Learning.

Oficina de las Naciones Unidas contra las drogas y el delito - UNODC (2010). La Relación Droga y Delito en Adolescentes Infractores de la Ley: La experiencia de Bolivia, Chile, Colombia, Perú y Uruguay. Recuperado de http://www.unodc.org/documents/peruandecuador/Publicaciones/ La_Relacion_Droga.pdf.

Penal de Lurigancho: los lujos y gollerías que tienen los reos (04 de abril, 2017). El Comercio. Recuperado de https://elcomercio.pe/lima/penallurigancho-lujos-gollerias-reos-412210.

Pérez, F (2011). Estudios sobre adicciones. Perfiles de drogodependientes $y$ eficacia del tratamiento en proyecto hombre burgos estudios sobre adicciones perfiles de drogodependientes y eficacia del tratamiento en proyectohombreburgos. España. Recuperadodehttp://proyectohombre. es/wp-content/uploads/2012/02/2011-Estudios-sobre-adicciones.pdf.

Revelan los nuevos métodos que se usan para ingresar drogas, celulares y armas a los penales. (30 de abril, 2017). La República. Recuperado de: https://larepublica.pe/sociedad/870770-descubren-nuevos-e- 
ingeniosos-metodos-para-ingresar-droga-celulares-y-armas-lospenales-del-pais.

Riveros, M (2004). Características Sociodemográficas y niveles de depresión en estudiantes ingresantes a la UNMSM a través de la escala CES-D. (Tesis de Maestria). Recuperado de http://cybertesis.unmsm.edu.pe/ bitstream/cybertesis/2726/1/riveros_qm.pdf.

Rodriguez, F., Bringas, C, De la Villa Moral, J., Pérez, B., y Estrada, C. (2012). Consumo de sustancias psicoactivas y delito: Análisis de la relación entre edad de inicio y reincidencia. International Journal of Psychological Research. Recuperada de http://www.scielo.org.co/pdf/ ijpr/v5n2/v5n2a07. Pdf.

Sánchez, X. (2011). Conductas Aditivas. En Jarne, A., Talarn, A. Manual de Psicopatología Clínica. Madrid: Herder.

Seger, D. (2010). Cocaine, metamfetamine, and MDMA abuse: the role and clinical importance of neuroadaptation. Recuperado de http://www. ncbi.nlm.nih.gov/pubmed/20849328.

Sun, W., Quizon P., y Zhu J (2016). Molecular Mechanism: ERK Signaling, Drug Addiction, and Behavioral Effects. Recuperado de http://www. ncbi.nlm.nih.gov/pubmed/26809997.

Valderrama, M (2014). Pasos para elaborar proyectos de investigación cientifica. Lima: San Marcos.

World Health Organization (2016). Psychoactive substances. Recuperado de http://www.who.int/substance_abuse/terminology /psychoactive_ substances/en/. 